\title{
Numerical Simulation of Nozzle Flow Field with Secondary Injection Thrust Vector Control
}

\author{
H.R. Noaman ${ }^{1, a}$, Tang Hai Bin ${ }^{1}$ and Elsayed Khalil ${ }^{2}$ \\ ${ }^{1}$ Beihang University, School of Astronautics, Beijing, China \\ ${ }^{2}$ Military Technical College, Aerospace Department, Cairo, Egypt
}

\begin{abstract}
Numerical simulations are performed to characterize the secondary injection thrust vector control. For this objective the following measurements were taken: considering the flow to be compressible and turbulent using Realizable $k-\varepsilon$ turbulence model accompanied by enhanced wall treatment, the comparison between the CFD results and the experimental results shows a very good agreement. Then a parametric study on injection mass flow rate (changing secondary stagnation pressure) with the same injection location and injection angle is done. The results stated that increasing the injectant mass flow rate lead to shock impingement from opposite wall at secondary stagnation pressure 1.4 of the primary stagnation pressure.
\end{abstract}

\section{Introduction}

The propulsion system not only provides a thrust to a rocket or any flying vehicle but also can introduce a control mechanism to correct the vehicle's trajectory and attitude by systems of thrust vector control (TVC). With the control of thrust vector direction, pitching, yawing and rolling moments can be accomplished on the aerial vehicle; there are many techniques to generate the deflection of the thrust vector such as the usage of flexible nozzle joints, gimbaled nozzles, jetavators, jet vanes/tabs, secondary injection, and etc. From the different ways to deflect thrust vector of a flying vehicle, Secondary Injection Thrust Vector Control (SITVC) has been applied successfully since 1960 's and is achieved by injecting secondary fluid to the supersonic flow inside the divergent part of the convergent-divergent nozzle. In contrast to mechanical thrust vector control systems, such as gimbaled nozzles, jet vanes/tabs, jetavators etc., that uses an actuation system to move the mechanical parts, SITVC does not use any movable parts and is governed by flow regulation, which minimize the losses of the axial thrust force during changing the thrust direction [1] The secondary injectant, (liquid or gas) can be supplied from the combustion chamber as a bleed or from a separate gas generator and it creates a complex flow field in the nozzle divergent part which contains not only a strong bow shock that creates asymmetry and a weak separation shock due to separation of the boundary layer upstream of the injector, but also downstream of the injector a Mach disk and reattachment region accompanied by recompression were created [2-4]. Figure1. [2] Schematically depicts the flow field structure inside the nozzle setup as a result of secondary injection. The causes of the deflection are as a result of the side force which produced by a combined effect of, a) interaction (induced) force, due to pressure rise along the wall, and b) jet reaction force, caused by the momentum

\footnotetext{
${ }^{a}$ Corresponding author : hossamnoaman84@outlook.com
} 
of the injecting secondary fluid [5]. As the secondary injection in the supersonic flow creates a complex flow field, many past researches focused on both analytical models such as Blast-wave analogy model[4] to characterize the flow field due to secondary injection and experimental tests with cold flow [6,7] and real static firing [8,9]. While analytical models deals only with very low injection flow rates and lack in general, the experimental tests of cold flow and static firing provides the main data of SITVC to be utilized for further analyses, but these tests gives only macroscopic performance predictions with high cost. On the other side the evolution of computational power and numerical methods, results in developing the computational Fluid Dynamics (CFD) to give a detailed microscopic description of flow properties. CFD became an attractive alternative to analytical models and a strong complimentary tool to experimental tests $[10,11]$.

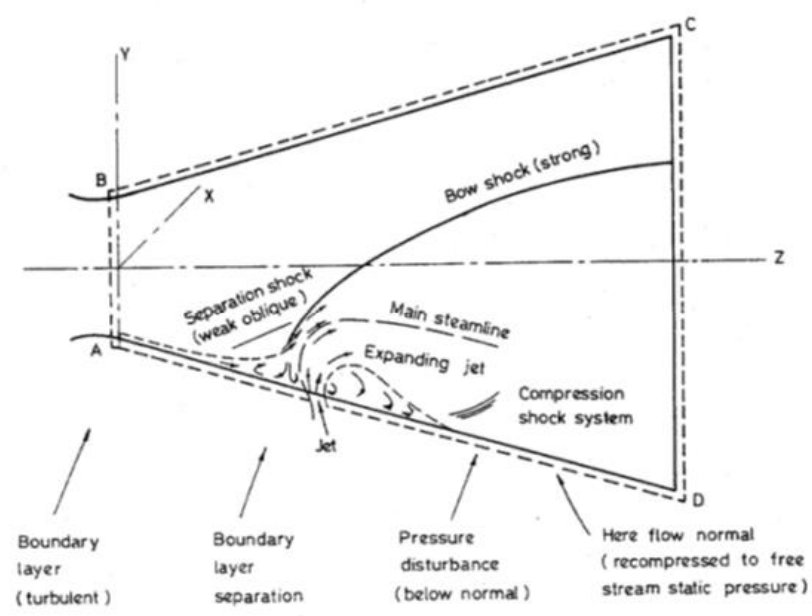

Figure 1. Flow field structure setup by secondary injection into primary nozzle flow [2]

In this paper the flow field investigation inside a nozzle in presence of secondary injection and analysis of SITVC performance parameters with variable injection mass flow rate (changing secondary stagnation pressure), for given injection location and injection angle were carried out.

\section{Numerical Methodology}

Numerical simulation of two dimensional flow field inside the nozzle used by Gushe [7] has been carried out with the aid of a steady Realizable $k-\varepsilon$ turbulence model with enhancement wall treatment. The solved equations are the two-dimensional, compressible, Reynolds-averaged Navier-Stokes equations, which were discretized in finite volume form on each of the quadrilateral control volumes. A second-order upwind scheme was chosen for the spatial discretization; the implicit density-based algorithm was used to solve the equation, and Roe-averaged flux difference splitting (Roe FDS) was chosen for the flux type, Ideal gas (air) was considered for analysis.

The first cell height inside wall nozzle was taken to achieve wall Y plus about 1 in the boundary layer as shown in figure 2. The first cell height estimation is estimated using skin friction coefficient $\left(C_{f}\right)$ for flat plate. Note that $\mathrm{x}$ is the distance from the nozzle inlet to injector location, where the separation of boundary layer occurs, and utilizing the definitions of wall Y plus and $U \tau$ the first cell height is predicted. Then it is validated with numerical simulation.

$$
C_{f}=0.0576\left(\mathrm{Re}_{\mathrm{x})}\right)^{-0.2}
$$




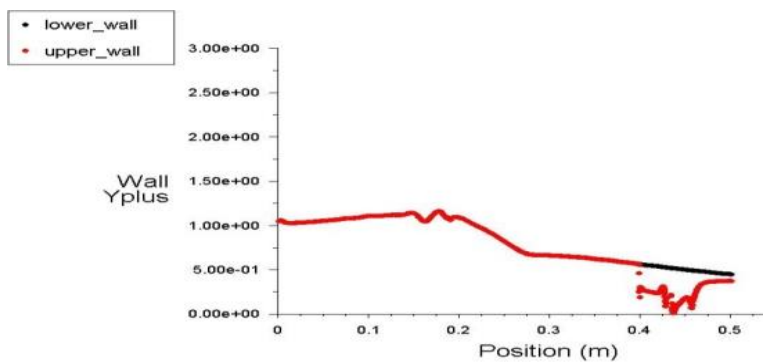

Figure 2. Wall Y plus-values along upper and lower wall

A typical structured grid system in the computational domain selected after grid independence study, where three grids with different aspects are considered as shown in Table1.

Table1. Computational grids for grid independence study

\begin{tabular}{|c|c|c|c|c|}
\hline NO & Grid size & $\begin{array}{c}\text { Injector } \\
\text { grid }\end{array}$ & $\begin{array}{c}\text { Height of } \\
\text { first grid }\end{array}$ & $\mathbf{y}^{+}$ \\
\hline 1 & $300 \times 690$ & $40 \times 75$ & 0.00002 in & $0.45-1$ \\
\hline 2 & $200 \times 640$ & $40 \times 75$ & 0.00002 in & $0.45-1$ \\
\hline 3 & $150 \times 400$ & $30 \times 75$ & 0.00004 in & $0.84-1$ \\
\hline
\end{tabular}

Grid independent study shows that grid 1 and 2 is a little better than grid 3 and for accuracy grid 2 was selected for the study.

The computational grid 2 are shown in figure 3.were the mesh around the injection slot and the walls were specially refined as shown in figure 4

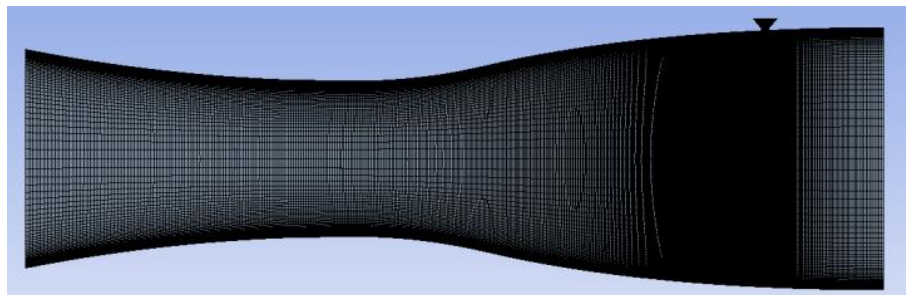

Figure 3. Computational structured Grid 2

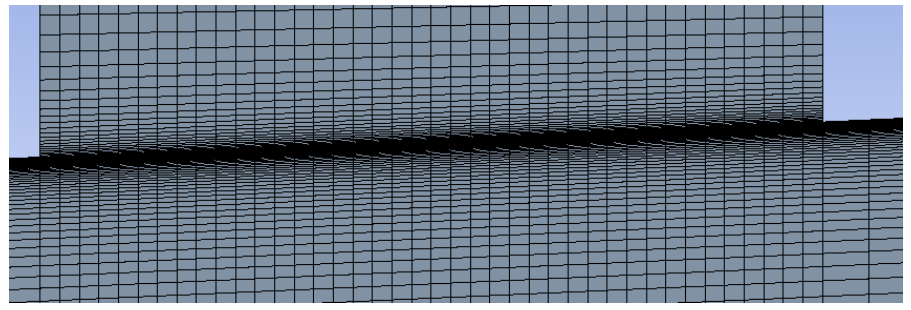

Figure 4. Close view at the injection grid 2

\subsection{Boundary conditions}

A pressure inlet boundary was used for nozzle inlet, secondary inlet and nozzle outlet where:

Inlet: Primary Stagnation Pressure, $\mathrm{P}_{\mathrm{op}}=100$ Psig, Primary Stagnation Temperature, $\mathrm{T}_{\mathrm{op}}=258.5 \mathrm{~K}$ 
Outlet: Primary Nozzle Exit Pressure, $\mathrm{P}_{\mathrm{ep}}=101068 \mathrm{~Pa}$

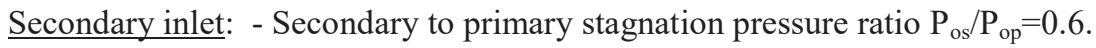

-Secondary injectant temperature $\mathrm{T}_{\mathrm{os}}=272.2 \mathrm{~K}$.

- Secondary injection slot to primary throat area ratio $\mathrm{A}_{\mathrm{s}} / \mathrm{A}^{*}=0.05$

-Injection location with respect to the primary flow Mach number Mp=1.904.

\subsection{Validation of the numerical simulation}

First numerical simulation of the flow field inside the nozzle used by Gushe [7] Without injection to calculate the primary axial thrust at no injection state and a comparison between the calculated Mach number [7] and the numerical one has been done as in figure 5.And shows a good accuracy with error less than $10 \%$.

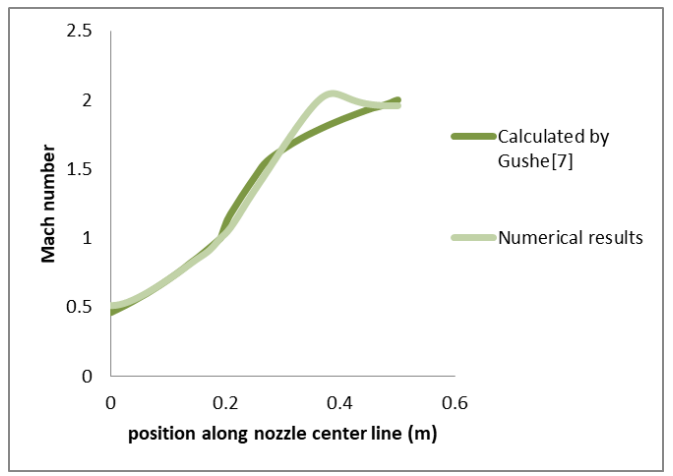

Figure 5. Comparison between calculated and numerical results for Mach number (no injection)

Then the simulation of the flow field of nozzle with the secondary injection slot carried out and a comparison between the experimental and numerical results has been done for the static pressure distribution along the upper wall where the injector have been installed using realizable $k-\varepsilon$ model, $k$ $\omega$ model and the shear stress transport (SST) $k$ - $\omega$ model it shows that all of the models behave similarly With an excellent accuracy in the unaffected zone and good accuracy in the high and low pressure zone less than $10 \%$ also a little difference between the experimental and numerical results at the boundary layer separation point figure 6 . Also the comparison for the static pressure distribution along lower wall (no injector) has been done and shows an excellent accuracy for all models figure 7 .

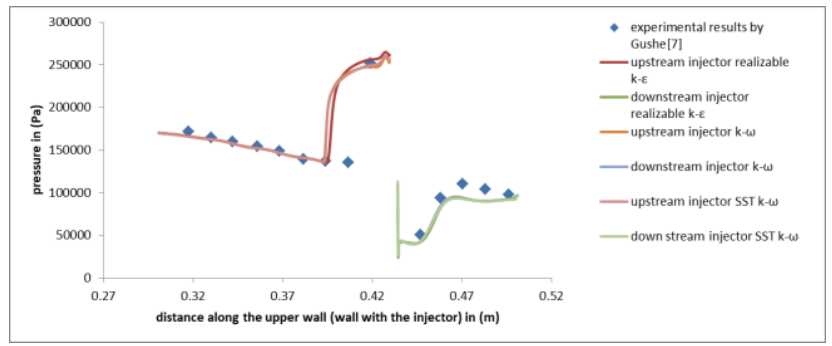

Figure 6.Comparison between Experimental and numerical results for pressure distribution upstream and downstream the injector on the upper wall using different turbulence models. 


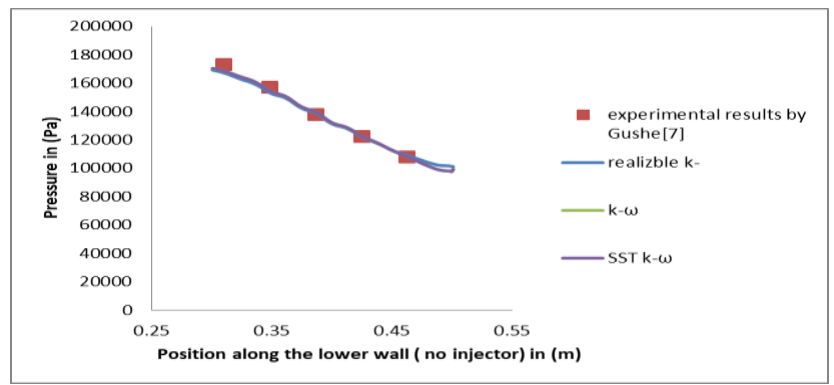

Figure7.Comparison between Experimental and numerical results for pressure distribution on the lower wall using different turbulence models

\subsection{SITVC Performance Parameters}

Secondary injection thrust vector control (SITVC) performance parameter investigated in present study includes [5-12]:

1-Axial Thrust Augmentation ATA\%

$$
A T A \%=\left(F_{P}-F_{P}^{o}\right) / F_{P}
$$

2-Thrust ratio is the ratio of the side thrust force to axial thrust force $F S / F p$ where:

*Side Thrust Fs:

$$
F_{s}=F_{i}+F_{j}
$$

$F_{i}$ is the interaction Force (Side-Thrust-Pressure Component).

$$
F_{i}=P A_{\text {upperwall }}-P A_{\text {lowerwall }}
$$

$F_{j}$ is the jet Reaction Force (Side-Thrust-Momentum Component).

$$
F_{j}=\dot{m}_{s} V_{s y}+\left(\left(P_{e s}-P_{a s}\right) A_{s}\right) \cos \beta_{i n j}
$$

*Primary axial thrust $F p$ :

$$
F_{P}=\dot{m}_{e} V_{x}+(P e-P a) A e
$$

3- System Specific Impulse Loss $\delta I s p$

$$
\delta I s p=I s p s y s-I s p^{\circ}
$$

4- Specific Impulse Ratio (Amplification Factor) AK is the ratio of the side specific impulse to main axial specific impulse and it determines the amount of fluid to be injected to have a specified side force [6].

$$
A K=I s p s / I s p^{\circ}
$$

where:

$$
\begin{aligned}
& I s p^{\circ}=F_{p} / \dot{m} \\
& I s p s=F_{s} / \dot{m}_{s}
\end{aligned}
$$




$$
\text { Ispsys }=\frac{\sqrt{F_{p}^{2}+F_{s}^{2}}}{\left(\dot{m}_{s}+\dot{m}_{p}\right) g}
$$

\section{Results and Discussion}

A parametric study on injection mass flow rate (changing secondary stagnation pressure) with the same injection location and injection angle is done as mass flow rate of the injectant varies directly with the injection total pressure.

$$
\dot{m}_{S}=\frac{A_{S} P_{O S}}{\sqrt{T_{O S}}} \sqrt{\frac{\gamma}{R}\left(\frac{2}{\gamma+1}\right)^{\frac{\gamma+1}{\gamma-1}}}
$$

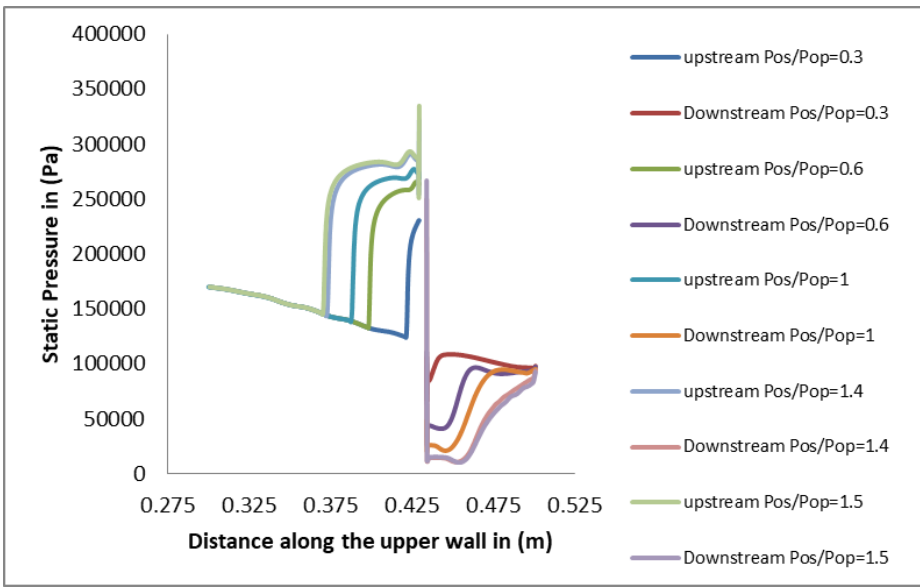

Figure 8. Effect of injection mass flow rate on injector wall static pressure distribution

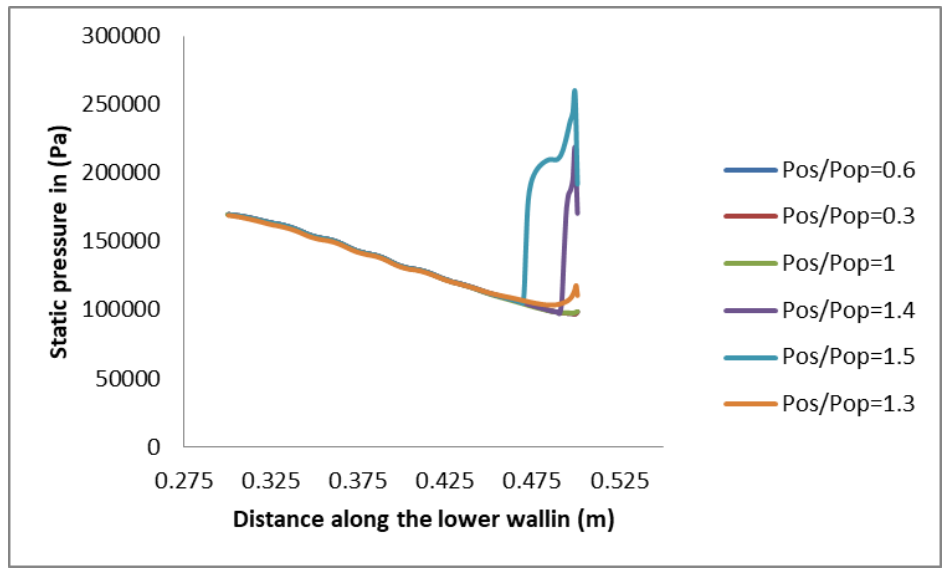

Figure 9. Effect of injection mass flow rate on lower wall static pressure distribution

The strength (shock angle) of the primary bow shock inside nozzle increases with the secondary mass flow rate (by increasing the secondary stagnation pressure) as shown in figure 8.

The origination point of the primary bow shock moves further upstream of the injector as the secondary mass flow rate increases (by increasing the secondary stagnation pressure). Leads to extended higher pressure region upstream of the injector as shown in figure 8 . 
Same as in primary bow shock, the strength of the separation increases as the secondary mass flow rate is increased (by increasing the secondary stagnation pressure). Stronger separation shock results into stronger injector upstream higher wall pressure region as shown in figure 8 .

Higher secondary mass flow rates (resulting from higher secondary stagnation pressure) also cause extended lower pressure regions downstream of the injector. As the injection mass flow rate is lowered, the reattachment point moves upstream on the primary nozzle wall in the aft section of the injector as shown in figure 9.

In figure 9 the effect of increasing the injectant mass flow rate on the lower wall it shows that no changes in the static pressure along the lower wall till reaching Pos/Pop=1.3 a very small shock impingement arises on the exit of the nozzle and when Pos/Pop increased to 1.4 and then 1.5 a significant shock impingement is shown leads to rise in the static pressure on the lower wall which effects the side force. The complex flow field with shock impingement setup by secondary injection inside primary supersonic flow was shown in Figure 10.

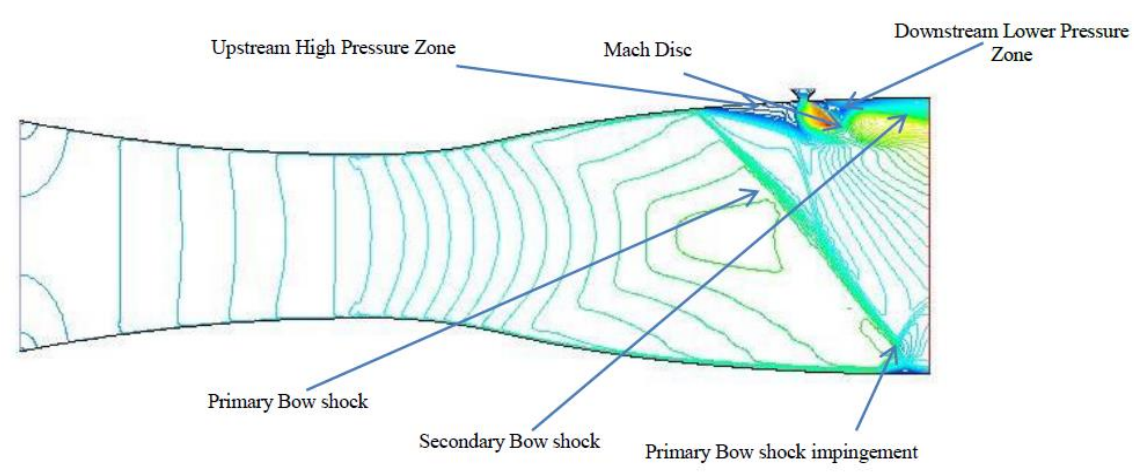

Figure 10. Flow field structure (Mach number contours) setup by secondary injection into primary nozzle flow.

The influence of injection stagnation pressure on the secondary injection performance parameters for a given injection location and angle of injection are shown in the figures from 11-14.

The observations are discussed as follows:

1 - Referring to figures 11-14, secondary mass flow rate is a function of secondary injection stagnation pressure. In other words, secondary mass flow rate varies directly with secondary injection stagnation pressure for a given injection area.

2 - Increasing the secondary mass flow rate (by increasing the secondary stagnation pressure), the axial thrust augmentation increases as can be observed in figure 14. Primary reason is addition \& subsequent adiabatic expansion of the injectant gas. Thrust augmentation is also a weak function of strength of the primary \& secondary bow shocks that deflect the primary flow direction in the primary nozzle. More augmentation to the axial thrust is resulted in case of stronger shocks.

3 - The thrust ratio increases linearly as the injectant mass flow rate increases (by increasing the secondary stagnation pressure) till reaching the critical secondary stagnation pressure where shock impingement occurs as observed in figure 11.

4 - The amplification factor in figure 12 increase linearly and rapidly for low mass flow rates for moderate and higher mass flow rates the rate of increase decreases till reaching the critical secondary stagnation pressure where shock impingement occurs as observed in figure 12. Amplification factor does not linearly depend on the secondary mass flow rate due to non-linearities of the governing factors. Amplification factor is a function of physical/flow properties of primary \& secondary fluids, injection location, angle of injection and primary nozzle geometry.

5 - Side thrust \& axial thrust augmentation is achieved at the cost of specific impulse. It can be observed in figures 13 that as the secondary mass flow rate increases (by increasing the secondary stagnation pressure), system specific impulse decreases almost linearly. The reason of this loss is the 
inefficient expansion of the injected gas. It is concluded that shock impingement surely affects the secondary specific impulse \& amplification factors.

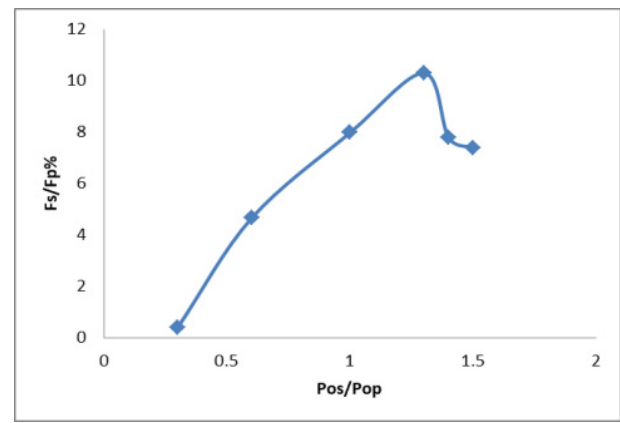

Figure11. Effect of secondary mass flow rate (by changing secondary stagnation pressure) on the thrust ratio

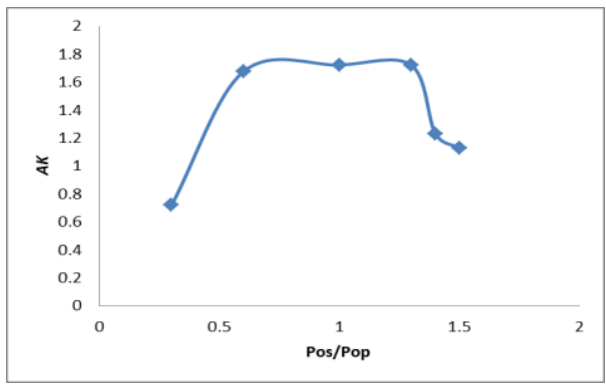

Figure12. Effect of secondary mass flow rate (by changing secondary stagnation pressure) on the amplification factor

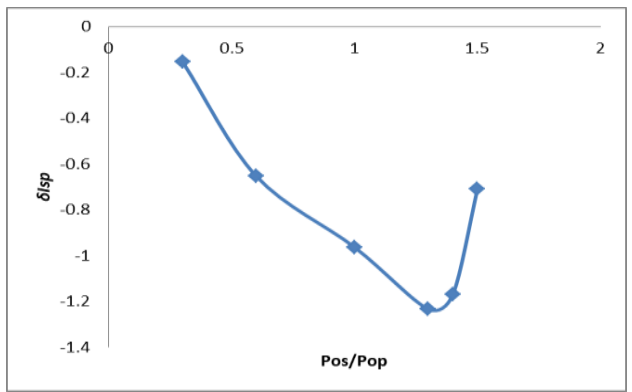

Figure13. Effect of secondary mass flow rate (by changing secondary stagnation pressure) on the specific impulse loss

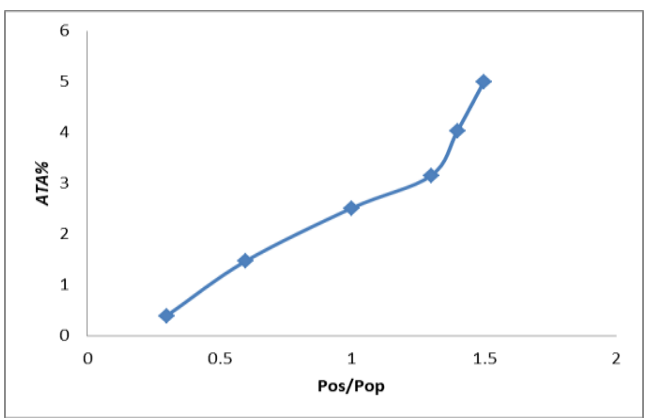


Figure14. Effect of secondary mass flow rate (by changing secondary stagnation pressure) on the axial thrust augmentation

\section{Conclusion}

The objective of the present paper was to investigate the performance and physics of SITVC. realizble $k-\varepsilon$ model with enhanced wall treatment used and compared to the experimental results to validate it. Performance parameters of SITVC system have been investigated with respect to different secondary mass flow rate. It was found that Injectant mass flow rate is a main parameter that can provide a wide range of operational flexibility \& controllability in SITVC systems and must be investigated to get the maximum injectant mass flow rate with safe injection to avoid shock impingement on the opposite side of the nozzle wall that leads to reduce net side force or in worst cases vectoring the system into undesired direction.

\section{Nomenclature}

\begin{tabular}{|c|c|}
\hline$A$ & Area $\left(\mathrm{m}^{2}\right)$ \\
\hline ATA\% & Axial thrust augmentation percentage \\
\hline$A K$ & Amplification factor \\
\hline$A_{s}$ & secondary injection area $\left(\mathrm{m}^{2}\right)$ \\
\hline$C_{f}$ & Skin friction coefficient \\
\hline$F$ & Force $(\mathrm{N})$ \\
\hline$F_{s}$ & Side force $(\mathrm{N})$ \\
\hline$F_{p}$ & Primary axial thrust $(\mathrm{N})$ \\
\hline$F_{p}{ }^{0}$ & Primary axial thrust without injection $(\mathrm{N})$ \\
\hline $\mathrm{M}_{\mathrm{p}}$ & $\begin{array}{l}\text { Injection location (in terms of primary flow axial Mach number corresponding to } \\
\text { injection point located on primary nozzle wall }\end{array}$ \\
\hline$\dot{\mathrm{m}}_{\mathrm{e}}$ & Total exit mass flow rate $(\mathrm{Kg} / \mathrm{s})$ \\
\hline$\dot{m}_{p}$ & Primary mass flow rate $(\mathrm{kg} / \mathrm{s})$ \\
\hline$\dot{m}_{s}$ & Secondary mass flow rate $(\mathrm{kg} / \mathrm{s})$ \\
\hline$P$ & Pressure $(\mathrm{Pa})$ \\
\hline$P_{e s}$ & Secondary exit pressure $(\mathrm{Pa})$ \\
\hline$P_{a s}$ & Ambient pressure at the secondary port before injection $(\mathrm{Pa})$ \\
\hline $\mathrm{P}_{\mathrm{os}}$ & Secondary stagnation pressure $(\mathrm{Pa})$ \\
\hline $\mathrm{P}_{\mathrm{op}}$ & Primary stagnation pressure $(\mathrm{Pa})$ \\
\hline $\mathrm{R}_{\mathrm{ex}}$ & Reynolds number at the distance $\mathrm{x}$ \\
\hline $\mathrm{T}_{\mathrm{os}}$ & Secondary stagnation temperature $(\mathrm{k})$ \\
\hline $\mathrm{U} \tau$ & Friction velocity $(\mathrm{m} / \mathrm{s})$ \\
\hline$V_{x}$ & Velocity along $\mathrm{x}-$ axis $(\mathrm{m} / \mathrm{s})$ \\
\hline$V_{s y}$ & Secondary gas velocity along y- direction $(\mathrm{m} / \mathrm{s})$ \\
\hline$I s p_{s}$ & Secondary specific impulse (sec) \\
\hline Ispsys & System specific impulse (sec) \\
\hline$I s p_{p}{ }^{o}$ & Primary specific impulse without injection (sec) \\
\hline$\delta I s p$ & Specific impulse loss \\
\hline$B_{i n j}$ & Wall angle at point of injection ( $\mathrm{deg}$ ) \\
\hline & Specific heat ratio \\
\hline
\end{tabular}

\section{References}

1. Sutton, G.W., "Rocket Propulsion Elements", 7th ed., John Wiley \& Sons, 2001.

2. Balu, R., "Analysis of Performance of a Hot Gas Injection Thrust Vector Control System", Journal of Propulsion andPower, Vol. 4, 1991, pp. 580-585. 
3. Newton, J.F., Spaid, F.M., "Interaction of Secondary Injectants and Rocket Exhausts for Thrust Vector Control", Journal of the American Rocket Society, August 1962, pp, 1203-1211.

4. Broadwell, J.E., "Analysis of Fluid Mechanics of Secondary Injection for Thrust Vector Control”, AIAA Journal, Vol. 1, No. 10, 1963, pp. 1067-1075.

5. Muhammad Usman Sadiq "Performance Analysis and Flowfield Characterization of Secondary Injection Thrust Vector Control (SITVC) for A 2DCD Nozzle" Msc. Thesis Faculty of the Viterbi School of Engineering University of Southern California, 2007.

6. Masuya, G., "Secondary Gas Injection into a Supersonic Conical Nozzle”, AIAA Journal, Vol. 15, No.3, 1977, pp. 301-302.

7. Guhse, R. D., "An Experimental Investigation of Thrust Vector Control by Secondary Injection" NASA CR-297 (1965).

8. Zeierman, I., Timnat, Y. M., "Full Control of Solid Propellant Rockets by Secondary Injection", Journal of Spacecraft and Rockets, Vol. 10, No. 3, 1973.

9. Inouye, T., "Experiments on Rocket Thrust Vector Control by Hot Gas Injection", Journal of Spacecraft and Rockets, Vol.3, No.4, pp. 737-739, 1966.

10. M.salehifar, M.Hojaji, A.Dartoomian, "CFD modeling for flow field characterization and performance analysis of HGITVC" applied thermal engineering 103,291-304,2016.

11. chuan Tian and Yijia Lu " turbulence models of separated flow in shock wave thrust vector nozzles" engineering applications of fluid mechanics vol7, no 2, 2013.

12. Erdem, E., Albayrak K., and Tinaztepe h. T., "Parametric Study of Secondary Gas Injection into a Conical Rocket Nozzle for Thrust Vectoring", Presented at $42^{\text {nd }}$ Joint Propulsion Conference, July 09-12, 2006. 